\title{
Zerstreute Aufmerksamkeit
}

\section{Empirischer Test eines Erklärungsmodells für die Nebenbeinutzung des Fernsehens}

Jens Wolling / Christoph Kuhlmann

Fernsehnutzung ist vielfach nur noch Nebenbeinutzung: Menschen essen, telefonieren, unterbalten sich mit anderen oder erledigen Hausarbeit, während gleichzeitig der Fernseher läuft. Der Beitrag forscht nach den Ursachen dieses Handelns, wobei die Autoren ein komplexes Erklärungsmodell entwickeln, in das Sozialisationsfaktoren, externe und interne Handlungsrestriktionen, die Bindung an das Fernsehen, Stimmungslagen, Qualitätswahrnehmungen sowie Motive und Leistungswahrnehmungen aus dem Uses and Gratifications-Ansatz eingehen. Durch multiple Regressionen wird nicht nur der Umfang der Nebenbeinutzung erklärt, sondern auch deren Modi, Inbalte und Situationen. Den stärksten Einfluss auf die Nebenbeinutzung haben drei Faktoren: Zum einen die positive Beurteilung spezifischer Leistungen des Nebenbeisehens - die insbesondere atmosphärischer Natur sind -, zum anderen die Fäbigkeit, mit den kognitiven Anforderungen durch die Paralleltätigkeit umzugehen, sowie drittens die Beurteilung der Qualität des Fernsehens. Die Varianz der übrigen erklärungskräftigen Variablen verdeutlicht die Vielfalt des Phänomens Nebenbeinutzung: Je nachdem, ob der Fokus der Analyse auf der Fernsehnutzung bei bestimmten Tätigkeiten oder auf der Nebenbeinutzung von spezifischen Angeboten liegt, erweisen sich unterschiedliche Gründe als bedeutsam.

Schlagwörter: Aufmerksamkeit, Nebenbeinutzung, Nebenbeifernsehen, Fernsehnutzung, Mediennutzung

\section{Einleitung: Ausgangssituation und Fragestellungen}

Die Fernsehnutzung erfährt seit einiger Zeit deutliche Veränderungen: Während sich die Nutzungszeit kaum verändert, treten verstärkt Nutzungsmodi auf, die früher dem Radio vorbehalten waren. Die Fernsehzuschauer gehen während des Fernsehkonsums anderen Beschäftigungen nach. Außerhalb der Prime Time widmet bereits mehr als die Hälfte der Zuschauer dem Fernsehen nicht mehr die volle Aufmerksamkeit ${ }^{1}$.

Theoretisch lenkt diese Beobachtung den Blick auf die Beziehung zwischen spezifischen Mediennutzungsaktivitäten und anderem Handeln. Dieses Handeln kann nicht nur Bedingung von Mediennutzung (etwa durch Beschaffung der technischen Ressourcen) oder Wirkung von Mediennutzung sein (wenn etwa Werbung zum Kauf führt), sondern auch funktionale Alternative ${ }^{2}$ oder Konkurrenz zur Mediennutzung: Konkurriert wird hier um Zeit und damit um Aufmerksamkeit ${ }^{3}$.

1 Diese und andere deskriptive Befunde zur Nebenbeinutzung des Fernsehens wurden an anderer Stelle ausführlich dargestellt (Kuhlmann \& Wolling 2004). Im gleichen Heft von M\&K haben sich auch Jäckel und Wollscheid mit ähnlichen Fragen beschäftigt. Die von ihnen präsentierten Befunde ergänzen unsere Ergebnisse - gerade auch unter methodischen Gesichtspunkten.

2 Funktionale Alternative kann Handeln etwa beim Mood Management sein, wenn beispielsweise Gartenarbeit die Stimmung zuverlässiger aufhellt als Fernsehkonsum.

3 Diese Perspektive lässt sich erweitern auf die Beziehungen zwischen Mediennutzung und Kognitionen (wann stört Nachdenken unsere Rezeptionsaufmerksamkeit?), aber auch zwischen Mediennutzung und interpersonaler Kommunikation und ebenso auf die Konkurrenz um Aufmerksamkeit zwischen verschiedenen Medien. 
Nebenbeisehen ist kein eindimensionales Konstrukt, sondern der Oberbegriff für verschiedene Formen der Paralleltätigkeit, bei denen neben der Fernsehnutzung andere Handlungen vollzogen werden, die ebenfalls Aufmerksamkeit beanspruchen. Diese Nutzungsmodi unterscheiden sich zum einen bezüglich des Sinneskanals in eine nur visuelle Fernsehnutzung (z. B. Telefonieren mit ausgeschaltetem Fernsehton) und eine vorwiegend auditive Nutzung (Haus- oder PC-Arbeit mit Hörkontakt zum Fernseher) sowie eine sozial orientierte Nebenbeinutzung, bei der zwar beide Kanäle wahrgenommen werden, ein Teil der Aufmerksamkeit aber von Interaktionen mit anwesenden anderen Akteuren beansprucht wird (geselliges Beisammensein und Essen mit laufendem Fernseher im Hintergrund) $)^{4}$.

Zum anderen variieren die Modi der Nebenbeinutzung in den Anteilen der Aufmerksamkeit für das Fernsehprogramm und die Begleittätigkeit. Dies hängt zentral vom Konzentrationsbedarf der Nicht-Fernsehtätigkeit ab und kann von weitgehend automatisierter Handarbeit bis zum Schreiben wissenschaftlicher Texte am Computer reichen. Der Grad der Aufmerksamkeit variiert nicht nur zwischen verschiedenen Paralleltätigkeiten, sondern häufig auch im Zeitverlauf bei ein und derselben Tätigkeit. Deshalb ist eine Differenzierung von Nutzungstypen entlang dieser Dimension schwierig. Zumal sich mit den in der Feldforschung einsetzbaren Methoden der Grad der Aufmerksamkeit nicht messen lässt: Kein Befragter vermag auch nur annähernd anzugeben, wie viel Prozent seiner Aufmerksamkeit dem Fernseher zuteil wird, wenn dieser zum Beispiel beim Essen läuft. Unter den nach Kanälen differenzierten Modi der Nebenbeinutzung dominiert in der Praxis das Nebenbeibören: Die Augen sind von anderen Tätigkeiten in Anspruch genommen, das Fernsehprogramm wird vor allem auditiv genutzt (Kuhlmann \& Wolling 2004). Hierfür eignen sich vor allem Fernsehformate, die auch ohne Bild befriedigend genutzt werden können, weil sich die Inhalte auch allein mittels der Toninhalte verstehen lassen. Dazu zählen primär Musikangebote, aber auch Formate wie Talk- und Gerichtsshows, bei denen im Bild wenig passiert, was zum Verständnis des Inhalts notwendig ist. Weitgehend irrelevant ist der Programminhalt dagegen, wenn das Fernsehen nur noch als Geräuschkulisse zur Vermeidung von Stille genutzt wird.

Alle Studien, die sich mit der Nebenbeinutzung des Fernsehens beschäftigen, zeigen, dass es sich um ein bedeutsames Phänomen handelt. ${ }^{5}$ Mittlerweile wissen wir auch einiges darüber, wie nebenbei ferngesehen wird. Wir haben aber keine systematischen Erkenntnisse über die Gründe dieser Nutzungsmuster. Warum verbringen die einen viel Zeit mit der Nebenbeinutzung, während die anderen fast überhaupt nicht nebenbei fernsehen? Gibt es unterschiedliche Erklärungen für die verschiedenen Modi der Nebenbeinutzung? Wird die Nebenbeinutzung bei unterschiedlichen Haupttätigkeiten auch durch unterschiedliche Erklärungsfaktoren beeinflusst? Lässt sich die Nebenbeinutzung bestimmter Angebote durch jeweils spezifische Variablen erklären, oder sind die Gründe inhaltsunspezifisch? Die schon bei der Deskription vorgenommene Differenzierung der Nebenbeinutzung in zeitlicher, sozialer und sachlicher Hinsicht wird hier wieder aufgegriffen, um die Frage nach den Ursachen differenziert beantworten zu können (Kuhlmann \& Wolling 2004: 387). Es geht also nicht nur darum, den Umfang der Nebenbeinutzung zu erklären, sondern es geht auch um die Erklärung der unterschiedlichen Modi der Nebenbeinutzung sowie der Nebenbeinutzung bestimmter Inhalte und der Nebenbeinutzung in bestimmten Situationen.

4 Die theoretische Unterscheidung bestätigte sich auch in einer Faktorenanalyse, in der diese Dimensionen identifiziert wurden (vgl. Kuhlmann \& Wolling 2004: 395).

5 Vgl. die Übersicht in Kuhlmann \& Wolling 2004. 


\section{Theoretische Grundlagen}

Für die Erklärung der Fernsehnutzung sind eine Reihe von Theorien entwickelt und eine Vielzahl von Modellen vorgeschlagen worden. Meistens handelt es sich dabei nicht um fernsehspezifische Ansätze, sondern um solche, die auch für die Erklärung der Nutzung anderer Medien (Printmedien, Hörfunk, Onlinemedien etc.) herangezogen werden können. Eine ausführliche Darstellung und kritische Diskussion dieser Theorien und Ansätze kann hier aus Platzgründen nicht erfolgen (vgl. dazu im Überblick Gehrau 2002; Hasebrink 2003; Meyen 2004). Da die meisten Theorien und Ansätze nicht auf bestimmte Inhalte oder Nutzungsmodi zugeschnitten oder beschränkt sind, bietet es sich an zu prüfen, ob sie für die Erklärung der Fernsehnebenbeinutzung nutzbar gemacht werden können. Da bislang kein Erklärungsmodell für die Nebenbeinutzung des Fernsehens vorliegt, wird auf mehrere der in Frage kommenden Ansätze zurückgegriffen und daraus ein Modell entwickelt (Grafik 1). Im Folgenden werden die verschiedenen Erklärungskomponenten dieses Modells näher erläutert.

\subsection{Soziodemographie, Sozialisation, Lebensstil, Restriktionen}

Die deskriptiven Auswertungen der Daten zur Nebenbeinutzung hatten ergeben, dass bei der Aufschlüsselung nach soziodemographischen Variablen teilweise erhebliche Unterschiede zwischen den Gruppen festzustellen waren. Insbesondere das Alter erwies sich immer wieder als bedeutsamer Faktor (Kuhlmann \& Wolling 2004). Die soziodemographischen Variablen können als Indikatoren für Sozialisationserfahrungen interpretiert und als Teil der sozialen Voraussetzungen der Mediennutzung angesehen werden (Katz, Blumler \& Gurevitch 1974). Neben solchen unspezifischen Indikatoren für soziale Erfahrungen sind auch spezifischere Indikatoren der Mediensozialisation zu berücksichtigen. Die wichtigste Instanz der Mediensozialisation sind zweifellos die Eltern. Wer in einem Haushalt aufgewachsen ist, in dem viel ferngesehen wurde, schaut vermutlich nicht nur selber viel fern (Jäckel 1997: 7), sondern neigt möglicherweise auch zu einer vermehrten Nebenbeinutzung, da durch die Fixierung auf das Fernsehen alternative Begleitmedien wie Radio oder Tonträger eine geringere Rolle spielen.

Neben dem sozialisationsbezogenen Bereich der sozialen Erfahrungen wirken sich sicherlich auch die aktuellen Bedingungen der Lebenssituation auf den Umfang und die Art der Nebenbeinutzung aus. Zu den Faktoren der sozialen Rahmenbedingungen gehört neben der beruflichen Einbindung und den Freizeitmöglichkeiten auch die Wohnsituation, insbesondere die Haushaltsausstattung mit Fernsehgeräten ist hier relevant. Die Nebenbeinutzung des Fernsehprogramms hängt von der Präsenz eines Fernsehgerätes zumindest in Hörweite ab: Je größer der Anteil der Räume in einer Wohnung, die keinen Fernseher aufweisen, desto seltener wird vermutlich nebenbei ferngesehen. Die Richtung einer möglichen Kausalität ist dabei unklar: Denkbar ist auch, dass intensive Nebenbeinutzer weitere Fernseher anschaffen bzw. angeschafft haben, um auch bei Tätigkeiten fernsehen zu können, die nicht im normalen Fernsehzimmer stattfinden.

Diese Überlegungen lassen sich theoretisch an zwei Konzepte anschließen: Einerseits an das Lebensstilkonzept von Rosengren (1996: 24 ff.), der die genannten Faktoren - soweit es sich um positionelle Merkmale (Alter, Geschlecht) handelt - unter der Rubrik Lebensweisen zusammenfasst, und - soweit es sich um individuell determinierte Merkmale (z. B. Fernsehausstattung) handelt - als Lebensstile bezeichnet.

Die Theorie der Rationalen Wahl stellt den zweiten Anknüpfungspunkt dar. Aus dieser theoretischen Perspektive handelt es sich bei den genannten Faktoren um Hand- 
lungsbedingungen, also um Restriktionen bzw. Optionen (Esser 1999: 52 ff.; Jäckel 1992; Vowe \& Wolling 2004: 78 f.). So ist beispielsweise die Berufstätigkeit im Allgemeinen eine Restriktion für die Nebenbeinutzung, denn wer berufstätig ist, kann während dieser Zeit meistens nicht nebenbei fernsehen. Generell ist zu erwarten: Wer (aus beruflichen Gründen) viel Zeit außer Haus verbringt, wird weniger Zeit haben, (nebenbei) fernzusehen. Anders hingegen verhält es sich mit der Beschäftigung zuhause. Insbesondere bei Hausarbeiten kann das Fernsehen eine angenehme Begleitung sein, aber auch eine zuhause ausgeübte Berufstätigkeit eröffnet zumindest die Möglichkeit zur Nebenbeinutzung.

Bei Restriktionen und Optionen kann zwischen externen und internen unterschieden werden. Externe Handlungsbedingungen sind solche, die der Akteur in der Umwelt wahrnimmt, wie z. B. die Anzahl der verfügbaren Fernsehgeräte. Die internen Handlungsbedingungen sind solche, die der Akteur in sich selbst verortet, wie z. B. seine Müdigkeit oder auch seine intellektuellen Fähigkeiten. Auch die im Rahmen von Kapazitätstheorien thematisierten kognitiven Potenziale, die bereits an anderer Stelle als möglicher Erklärungsansatz benannt wurden (Kuhlmann \& Wolling 2004: 389), können als interne Restriktionen interpretiert werden.

\subsection{Motivationale Ansätze: Uses and Gratifications}

Wenn im Rahmen der Medien- und speziell der Fernsehnutzungsforschung theoretisch fundierte Forschung betrieben wird, dann spielt der Uses and Gratifications Approach (vgl. Schenk 2002; Vorderer 1992; Rubin 2002), der später im Wert-Erwartungsmodell und dem Diskrepanzmodell (Palmgreen, Wenner \& Rayburn 1981; Palmgreen \& Rayburn 1982; Vowe \& Wolling 2001) weiterentwickelt wurde, eine herausragende Rolle. Wir haben die gesuchten Gratifikationen in Form von allgemeinen kommunikationsbezogenen Nutzungsmotiven erhoben und zum anderen die erbaltenen Gratifikationen direkt als wahrgenommene Leistungen des Fernsehens ermittelt.

Bei der Suche nach Motiven von Fernsehnutzern, ${ }^{6}$ die ihre Nebenbeinutzung erklären können, liegt es nahe, sich zunächst denjenigen Nutzungsmotiven zuzuwenden, die auch zur Erklärung aufmerksamer Fernsehnutzung herangezogen werden. Hierfür kann auf zahlreiche theoretische Systematisierungsvorschläge und empirisch getestete Aufstellungen der verschiedenen kommunikationsbezogenen Motive zurückgegriffen werden (McGuire 1974; Frank \& Greenberg 1980; McQuail, Blumler \& Brown 1972). An diese Ausarbeitungen konnte angeknüpft werden, denn einige Modi der Nebenbeinutzung - wie etwa der häufig auftretende auditive Modus des Fernsehbörens - können durch Motive - wie etwa das nach aktueller Information ${ }^{7}$ oder Eskapismus - möglicherweise genauso gut erklärt werden wie das aufmerksame Fernsehen. Das Fluchtmotiv könnte einen positiven Effekt auf den Umfang der Nebenbeinutzung haben, da eine solche

6 Da Fernsehen zunehmend Fernbören wird, sprechen wir nicht mehr vom Zuschauer sondern vom Nutzer.

7 Das Bedürfnis, ständig über das aktuelle Weltgeschehen auf dem Laufenden zu sein, kann zur Hintergrundnutzung des Fernsehens führen, indem zum Beispiel den regelmäßigen Nachrichtensendungen kurz die Aufmerksamkeit geschenkt wird, oder weil damit gerechnet wird, dass bei außergewöhnlichen Ereignissen (Extremereignissen) wie beispielsweise den Terroranschlägen vom 11. September das Fernsehen das Programm ändert und live über die Geschehnisse berichtet. Die große Bedeutung, die das Fernsehen für die Information über solche Extremereignisse hat, verdeutlichen Ergebnisse von Emmer et al. (2002). 
Fernsehnutzung von den Alltagsproblemen ablenken kann. Der nebenbei laufende Fernseher vertreibt die (auch Einsamkeitsgefühle nährende) Stille im Raum und stört mögliche Prozesse der (negativen) Selbstreflexion. Auch das Motiv der Anregungssuche sollte die Nebenbeinutzung positiv beeinflussen. Schließlich liefert das Fernsehangebot einen permanenten Fluss von Reizen, die den Wunsch nach Anregungen befriedigen könnten. Insbesondere bei anregungsarmen Tätigkeiten - etwa der Hausarbeit - legt ein derartiges Motiv die Nebenbeinutzung nahe.

Neben diesen klassischen Motiven sind jedoch auch solche denkbar, die bei der Nebenbeinutzung eine besondere Rolle spielen, etwa der Wunsch nach Vertreibung der Stille durch eine Geräuschkulisse oder die sinnvolle Organisation der Zeit. Für das Organisationsmotiv sind allerdings gegenläufige Hypothesen plausibel: Das Bedürfnis nach Zeitorganisation kann zu einer systematischen Trennung verschiedener Aktivitäten, aber auch zu einer geplanten parallelen Ausübung verschiedener Tätigkeiten führen ${ }^{8}$.

\subsection{Subjektive Qualitätsauswabl}

Ein Kritikpunkt am Uses and Gratifications Approach ist seine Inhaltsvergessenheit (Vorderer 1992: 32). Gemeint ist damit, dass die Erklärung für die Nutzung der Angebote allein bei den Rezipienten gesucht wird, die Inhalte und Angebotseigenschaften jedoch vernachlässigt werden. Dieses Defizit soll durch die Berücksichtigung subjektiver Qualitätswahrnehmungen kompensiert werden (vgl. Wolling 2004).

Die Qualitätswahrnehmungen können in mehrfacher Hinsicht für die Nebenbeinutzung des Fernsehens von Bedeutung sein. Zum einen kann die Wahrnehmung der aktuellen Qualität des Angebots einen Effekt auf die Nebenbeinutzung haben, zum anderen können sich aber auch wahrgenommene Qualitätsveränderungen auswirken. Wenn das Fernsehangebot als immer schlechter werdend erlebt wird, könnte dies erklären, warum die Nutzer ihm keine volle Aufmerksamkeit mehr widmen mögen. Umgekehrt ist es aber auch denkbar, dass das Fernsehangebot deswegen vermehrt nebenbei genutzt wird, weil man von dem Angebot so begeistert ist, dass man nichts verpassen möchte und es deshalb auch (nebenbei) nutzt, wenn andere Tätigkeiten anstehen.

Darüber hinaus könnte auch die wahrgenommene Qualität des Radios die Fernsehnebenbeinutzung beeinflussen. Eine solche Beziehung ist plausibel, weil das Radio das Hauptkonkurrenzmedium der Nebenbeinutzung darstellt. Wenn die Qualität des Radioprogramms als schlecht oder als verschlechtert erlebt wird, dann ist mit einer höheren Nebenbeinutzung des Fernsehens zu rechnen.

\subsection{Bindung an das Fernseben}

In der Studie Massenkommunikation wird schon seit den 70er Jahren die Bindung an die Massenmedien erhoben, indem ermittelt wird, wie sehr das jeweilige Medium vermisst würde, wenn es nicht mehr zur Verfügung stünde (Ridder et al. 2002: 24 f.). Auch im englischen Sprachraum wird diesem Konzept unter dem Stichwort Media Dependency Aufmerksamkeit geschenkt. Hier spielt es jedoch vor allem als Erklärungsvariable in Medienwirkungsprozessen eine Rolle (Ball-Rokeach \& DeFleur 1976; Becker \&

8 Dahinter stecken wahrscheinlich zwei unterschiedliche Konzepte: Das Bedürfnis nach Organisation einzelner Zeiteinheiten dürfte eher zur Trennung der Tätigkeiten, das Bedürfnis nach Organisation eines knappen Zeithaushaltes eher zur parallelen Ausübung führen. Diese Differenzierung konnte allerdings in der empirischen Studie noch nicht berücksichtigt werden. 
Whitney 1980) oder fungiert als abhängige Variable in der Nutzungsforschung (Wenner 1982). Es erscheint sinnvoll, die TV-Bindung im Rahmen dieser Studie zu berücksichtigen, da bei einer starken Bindung an das Fernsehen mit einer intensiven Nutzung - auch als Nebenbeimedium - zu rechnen ist.

\subsection{Stimmungslagen}

Die Theorie des Mood Management besagt, dass Stimmungen und der Wunsch, sie zu optimieren, die Fernsehnutzung beeinflussen. Die Rezipienten reagieren mit der Auswahl von Medien und spezifischen Medienangeboten auf ihre jeweilige Stimmung, um diese so durch die Nutzung adäquater Angebote zu beeinflussen (Zillmann, Hezel \& Medoff 1980, Zillmann 1994). Die Theorie basiert auf der Annahme, dass Menschen hedonistische Wesen sind, die einerseits bemüht sind, positive Stimmungslagen zu erreichen, und andererseits gleichzeitig ein mittleres Erregungsniveau anstreben. Insbesondere dem Fernsehen sowie der Nutzung von Musik (Wünsch 2001) wird die Fähigkeit zugeschrieben, stimmungsregulierend wirken zu können.

Die vorliegenden empirischen Befunde bestätigen die Theorie nur teilweise. Vor allem in experimentellen Laborsituationen zeigt sich, dass Rezipienten gemäß ihrer aktuellen Stimmung Medienangebote auswählen. Diese Vorgehensweise ist jedoch stark kritisiert worden (Schmitz \& Lewandrowski 1993). In den wenigen vorliegenden Feldstudien sind die Befunde nicht eindeutig. Donsbach und Tasche (1999) kommen zu dem Ergebnis, dass das Auswahlverhalten sich zwar an den individuellen Stimmungen orientiert, die Nutzung der Angebote dann aber nicht unbedingt zu der erhofften Stimmungsregulierung führt. Die Befunde legen die Interpretation nahe, dass nicht die tatsächliche Veränderung, sondern vielmehr der erwartete Effekt auf die Befindlichkeit für die Nutzer von Bedeutung ist. Dieses Ergebnis wiederum spricht dafür, dass es sich bei der stimmungsbasierten Auswahl um ein in hohem Maße habitualisiertes Verhalten handelt. Wenn dies der Fall ist, dann sollte sich auch dann, wenn man von einzelnen Nutzungssituationen abstrahiert, ein Zusammenhang zwischen Stimmung und Mediennutzung zeigen. Je nachdem, in welchen Stimmungslagen sich Personen häufiger befinden, sollte ihr Rezeptionsverhalten sich unterscheiden.

Es erscheint plausibel, dass die Rezipienten von den Begleitgeräuschen des Fernsehens einen regulierenden Effekt erwarten, etwa zur Aufhellung der Stimmung während lästiger Arbeiten. Vor allem dann, wenn negative Stimmungen bei geringem Erregungsniveau (Deprimiertheit, Energielosigkeit) häufig vorkommen, sollten die Hintergrundgeräusche der Nebenbeinutzung („virtueller Mitbewohner") als angenehm bzw. Stille in der Wohnung als unangenehm erlebt werden. Bei vorherrschend positiven Stimmungslagen mit niedrigem Erregungsniveau (Besinnlichkeit, Ruhe) ist eher mit einer geringfügigen Nebenbeinutzung zu rechnen, da in diesem Fall Hintergrundgeräusche vermutlich eher störend wirken. Welche Effekte von Stimmungslagen mit hohem Arousal und negativer Bewertung (Ärger, Erregtheit) bzw. hohem Arousal und positiver Bewertung (Aktivität, gehobene Stimmung) auf die Nebenbeinutzung ausgehen könnten, lässt sich nur schwer vorhersagen. 


\subsection{Indirekte Effekte}

Möglicherweise wirken sich die Stimmungslagen jedoch nicht nur direkt auf die Nebenbeinutzung aus, sondern auch indirekt über die gesuchten Gratifikationen (Motive). Wenn Stimmungen relativ stabil sind (Zillmann 2004) und bei unterschiedlichen Personen verschiedene Stimmungslagen dominieren, kann sich ein solcher Zusammenhang ergeben. Aber nicht nur bei den Stimmungslagen erscheint es plausibel, dass sich indirekte Effekte ergeben. Zu erwarten ist beispielsweise auch, dass sich die Bindung an das Fernsehen auf die Motivationsstärke auswirkt, dass die Leistungsbewertungen einen Einfluss auf die Qualitätswahrnehmungen haben und dass sich die Sozialisationsfaktoren auch auf Restriktionen, Stimmungslagen, die Motive und die TV-Bindung auswirken. Die hier vermuteten Zusammenhänge sind in Grafik 1 im Überblick dargestellt.

\section{Grafik 1: Erklärungsmodell der Nebenbeinutzung des Fernsebens}

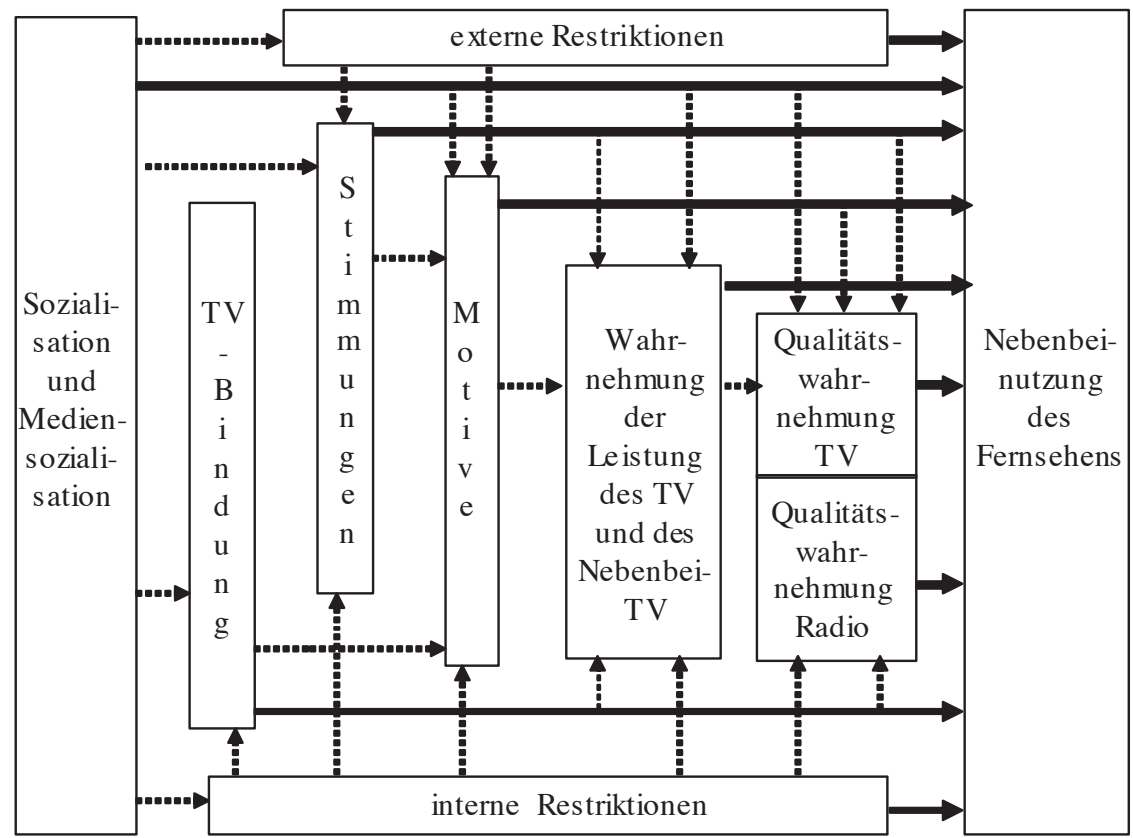

Im Modell wird zum einen eine bestimmte Kausalitätsrichtung zwischen den verschiedenen Variablen angenommen; zum anderen wird vermutet, dass die verschiedenen unabhängigen Variablen nicht gleichberechtigt nebeneinander stehen, sondern dass Differenzierungen vorgenommen werden sollten. Die Unterscheidung von unabhängigen und abhängigen Variablen ist dem zugrunde liegenden Erkenntnisinteresse geschuldet. Mit dieser Einteilung soll nicht angedeutet werden, dass es sich grundsätzlich um einseitige Kausalzusammenhänge handelt. Gerade die Qualitäts- und Leistungswahrnehmungen sind natürlich nicht nur Ursache, sondern auch Ergebnis der Nutzung. Bei der Anordnung der unabhängigen Variablen wurde versucht, dieser Umkehrbarkeit Rechnung zu tragen. Die weiter links stehenden Variablen werden als stabiler und durch 
die Nebenbeinutzung weniger beeinflussbar angesehen als diejenigen, die weiter rechts - näher an der abhängigen Variablen - angeordnet wurden.

\section{Datenerhebungsmethoden und Operationalisierungen}

Die Datenerhebung wurde mittels einer schriftlichen, vollstandardisierten Befragung auf Basis einer Quotenstichprobe von 302 Befragten durchgeführt. Mit dem Fragebogen erhielten die Befragten ein Tagebuch, in dem sie an je einem Wochen- und Wochenendtag in Viertelstundenintervallen ihre Tätigkeiten protokollieren sollten. ${ }^{9} 51$ Prozent der Befragten sind weiblich und 49 Prozent männlich. Das Durchschnittsalter beträgt 46 Jahre. Die jüngsten Befragten sind 15 und die ältesten 82 Jahre alt. 51 Prozent der Befragten haben maximal Hauptschulabschluss, 27 Prozent absolvierten die Realschule, 11 Prozent haben Abitur und weitere 10 Prozent haben darüber hinaus die Universität besucht.

Die verschiedenen Merkmalsgruppen wurden im Fragebogen durch umfangreiche Itembatterien operationalisiert. Die Konstrukte wurden dabei soweit möglich durch mehrere Items abgebildet. In diesem Fall ist es sinnvoll, mittels Faktoranalysen die Vielzahl der Items wieder auf die zugrunde liegenden Dimensionen zu reduzieren. Damit wird nicht nur Komplexität reduziert, sondern auch geprüft, ob die gewählten Items tatsächlich diejenigen Konstrukte abbilden, die theoretisch erwartet werden. Um Dopplungen zu vermeiden, verbinden wir im Folgenden die Präsentation der Fragebogenitems mit den Ergebnissen dieser Faktorenanalysen und präsentieren auch bereits erste deskriptive Befunde.

\subsection{Abhängige Variable: Dauer der Nebenbeinutzung}

Eine annähernd perfekte Messung von Umfang und Art der Paralleltätigkeiten zum Fernsehen würde eine permanente Videoüberwachung der Nutzer voraussetzen - und hätte als Folge mit Problemen der Reaktivität zu kämpfen. Auch der einseitige Rückgriff auf klassische Befragungsmodelle ist problematisch, da sie von den Befragten einen sehr hohen Grad an Selbstbeobachtung fordern. Deshalb wurden in der Untersuchung zwei unterschiedliche Verfahren eingesetzt:

Befragung: Im Fragebogen wurde im Anschluss an die Fragen nach dem durchschnittlichen Fernsehkonsum an Wochentagen und Wochenenden um eine Schätzung gebeten, wie groß jeweils der Anteil der Fernsehzeit ist, bei der gleichzeitig einer anderen Tätigkeit nachgegangen wird. Um die Selbstbeobachtungskompetenz der Befragten nicht zu überfordern, wurde hier eine recht grobe Skala mit den Ausprägungen 0, 25, 50, 75 und 100 Prozent vorgegeben.

Tagebuch: Die Befragten wurden gebeten, für je einen Wochentag und einen Wochenendtag ein Tagebuch auszufüllen, in dem in Viertelstundenintervallen Mediennutzung und andere Tätigkeiten protokolliert werden sollten. Auf der Basis dieser Daten ließ sich dann ebenfalls hochrechnen, wie viel Zeit täglich mit der Nebenbeinutzung des Fernsehens verbracht wird.

Wie Tabelle 1 zeigt, führen beide Verfahren zu teilweise unterschiedlichen Ergebnissen. Während wochentags noch fast identische Ergebnisse erzielt werden, ist der Umfang der Nebenbeinutzung laut Tagebuch am Wochenende wesentlich höher. Gleichzeitig korrelieren die Wochenendwerte aber wesentlich höher miteinander als die Werte

9 Weitere ausführliche Informationen zur Methode finden sich bei Kuhlmann \& Wolling (2004). 
Tabelle 1: Umfang der Nebenbeinutzung in Fragebogen und Tagebuch

\begin{tabular}{lllll}
\hline & Fragebogen & Tagebuch & Korrelation & Index \\
\hline Wochentag & $50 \mathrm{~min}$ & $52 \mathrm{~min}$ & $.26^{* * *}$ & \\
Wochenende & $55 \mathrm{~min}$ & $81 \mathrm{~min}$ & $.45^{* * *}$ & \\
Gesamt & $52 \mathrm{~min}$ & $61 \mathrm{~min}$ & $.44^{* * *}$ & $56 \mathrm{~min}$ \\
\hline
\end{tabular}

für Wochentage. Das kann mehrere Ursachen haben. Zum Teil sind diese Unterschiede sicherlich methodisch bedingt. Die erhöhte Selbstaufmerksamkeit beim Ausfüllen eines Tagebuchs führt vermutlich zu einem exakteren Ergebnis, von daher wären die Tagebuchdaten vorzuziehen. Andererseits ist aber davon auszugehen, dass die intraindividuelle Varianz der Nebenbeinutzung tatsächlich recht hoch ist und deswegen die an dem einen Tag per Tagebuch gemessenen Werte von der durchschnittlichen Nutzung deutlich abweichen. Dass diese Varianz vor allem an Wochentagen deutlicher ausfällt, erscheint plausibel. Aus diesem Grund war die ermittelte geringere Korrelation zu erwarten. Um die Nachteile der jeweiligen Methode zu kompensieren, wurden die beiden Messungen als Indikator für den Umfang der Nebenbeinutzung zu einem Gesamtindex zusammengefasst.

\subsection{Abbängige Variablen: Modi der Nebenbeinutzung}

Die postulierten Dimensionen der audio-, video- und sozialorientierten Nebenbeinutzung wurden durch eine Reihe von Items operationalisiert, die mittels einer Faktorenanalyse wieder auf die Ursprungsdimensionen zurückgeführt werden konnten. ${ }^{10}$ Die videoorientierte Nutzung (Fernsehen ohne Ton) tritt so selten auf, dass wir sie hier ausklammern. Die audioorientierte und die sozialorientierte Nutzung wurden durch Indizes der Faktoritems abgebildet. ${ }^{11}$

\subsection{Abhängige Variablen: Tätigkeitsbezogene Indikatoren der Nebenbeinutzung}

Mittels einer 5er-Skala (nie, selten, ab und zu, oft, sehr oft) wurde erfragt, wie häufig die Befragten bei bestimmten Tätigkeiten den Fernseher laufen lassen. Diese Tätigkeiten lassen sich mittels einer Faktorenanalyse sinnvoll auf zwei Faktoren reduzieren: Pflichtaufgaben einerseits ${ }^{12}$ und Freizeittätigkeiten andererseits ${ }^{13}$, die zusammen $52 \%$ der Varianz erklären. ${ }^{14}$

10 Die Ergebnisse der Faktorenanalyse wurden bereits veröffentlicht, vgl. Kuhlmann \& Wolling 2004: $393 \mathrm{f}$.

11 Audioorientierte Nebenbeinutzung: Items „Hin und wieder schaue ich kurz hin, wenn in der Sendung etwas Interessantes kommt.“; „Der Fernseher läuft und ich passe gar nicht richtig auf, was kommt.“; „Ich schaue zwar nicht hin, höre aber aufmerksam zu.“; „Der Fernseher ist wie eine Geräuschkulisse, ich kriege eigentlich nicht mit, was da läuft."; Sozialorientierte Nebenbeinutzung: Items „Wenn mich jemand besuchen kommt, schalte ich den Fernseher aus.“; „Ich unterhalte mich mit anderen, während der Fernseher läuft."

12 Eigenwert 3,9; Items (Faktorladungen): Essen (.84), Kochen (.83), Hausarbeit (.59), Körperpflege (.55).

13 Eigenwert 1,3; Items (Faktorladungen): Spiel (.76), Lesen (.65), Sport (.63), Gespräche (.63), Hobby (.60). Nur die Beschäftigung Hobby hat eine nennenswerte Nebenladung (.43).

14 Ausgeschlossen wurden die Items Beruf und Telefon wegen hoher Doppelladungen, die sich aber erklären lassen: Telefonieren kann beruflich bedingt und damit Pflichtaufgabe oder aber 


\subsection{Abbängige Variablen: Inhalte der Nebenbeinutzung}

Die Inhalte der nebenbei genutzten Programme wurden mit einem zweistufigen Verfahren erhoben: Zunächst wurde für eine umfangreiche Liste von Genres mittels einer 4er-Skala (nie, selten, gelegentlich, häufig) ermittelt, wie häufig diese Genres überhaupt genutzt werden. Für alle zumindest gelegentlich genutzten Genres wurde dann mit derselben Skala gefragt, „wie häufig es vorkommt, dass Sie gleichzeitig etwas anderes tun, wenn eine solche Sendung läuft". Die Häufigkeit der Nebenbeinutzung bestimmter Inhalte liegt damit bei jedem Genre für eine andere Gruppe von Befragten vor (nämlich die jeweils gelegentlichen oder häufigen Nutzer des Genres). Das macht eine Faktorenanalyse zur Reduktion der Itembatterie problematisch. Deshalb haben wir aus den Fragen zur allgemeinen Nutzung und zur Nebenbeinutzung der Genres durch Multiplikation der beiden Variablen einen Index erstellt, der den Umfang der Nebenbeinutzung eines Genres abbildet (Tabelle 2). In den Feldern der Tabelle sind die Werte der neu gebildeten Variablen dargestellt (Werte 0-6):

Tabelle 2: Berechnung der Indexwerte für die Nebenbeinutzung von Fernsehgenres

\begin{tabular}{llcccc}
\hline & & \multicolumn{4}{c}{ Nebenbeinutzung des Genres } \\
& & nie $(0)$ & selten (1) & gelegentlich (2) & häufig (3) \\
\hline \multirow{2}{*}{ Generelle } & nie (0) & 0 & 0 & 0 & 0 \\
Nutzung & selten (0) & 0 & 0 & 0 & 0 \\
des Genres & gelegentlich (1) & 0 & 1 & 2 & 3 \\
& häufig (2) & 0 & 2 & 4 & 6 \\
\hline
\end{tabular}

Auf der Basis dieser Indizes lassen sich die Fernsehgenres mittels einer Faktorenanalyse zu vier Faktoren zusammenfassen, die 59\% der Varianz erklären ${ }^{15}:$ Im ersten Faktor ${ }^{16}$ versammelt sich die Nebenbeinutzung von Genres der leichten Unterhaltung und des Infotainments, die sich vor allem durch zwei Merkmale auszeichnen: a) Sie lassen sich wie ein Radioprogramm nutzen, da die meisten Inhalte auf dem Audiokanal vermittelt werden; b) sie werden überwiegend nachmittags und am Vorabend ausgestrahlt. Der zweite Faktor ${ }^{17}$ enthält ausschließlich klassische Informationsangebote. Die Items $\mathrm{Mu}$ sik und Comedy im dritten Faktor ${ }^{18}$ verbindet, dass es sich dabei um fragmentierte Un-

auch Freizeitbeschäftigung sein. Berufliche Tätigkeit kann ohne Präsenz eines Fernsehers am Arbeitsplatz, aber auch daheim erfolgen.

15 Wegen hoher Doppelladungen ausgeschlossen wurden die Items Spielfilme, wöchentliche Serien und Quizshows. Die ersten zwei Items bezeichnen Formate mit Spielhandlungen, bei denen für den Befragten offen bleiben musste, welchen Charakter ihr Inhalt jeweils hat. Das gleiche gilt für Quizshows, bei denen Unterhaltung oder Information im Vordergrund stehen kann. Ferner wurden folgende Genres aus der Analyse ausgeschlossen, die aus verschiedenen Gründen nicht in die Systematik passen: Kindersendungen (diese werden von erwachsenen Befragten wohl häufig unfreiwillig nebenbei verfolgt), Frühstücksfernsehen (fällt aufgrund der Sendezeit aus dem Rahmen), Teleshopping-Sendungen, Werbung und Erotik-Sendungen.

16 Eigenwert 3,6; Items (Faktorladungen): Talkshows (.84), Boulevardmagazine (.68), tägliche Serien (.66), Gerichtssendungen (.65), Reality-Sendungen (.64).

17 Eigenwert 2,1; Items (Faktorladungen): Natursendungen (.77), Dokumentarfilme (.76), politische Magazine (.75), Nachrichten (.63).

18 Eigenwert 1,1; Items (Faktorladungen): Musiksendungen (.85), Comedy (.64). 
terbaltung handelt, schnell geschnittene kurze Szenen in den Comedys und Videoclips wechseln sich in rascher Folge ab, längerfristige Aufmerksamkeit ist nicht erforderlich. Die Genres Sport und Krimi des vierten Faktors ${ }^{19}$ weisen beide einzelne spannende Höhepunkte (Mord, Elfmeter) auf, bei denen der Rezipient vielleicht kurz mit voller Aufmerksamkeit zuschaut, um sich dann wieder der Haupttätigkeit zuzuwenden.

\subsection{Unabhängige Variablen: Sozialisation und Mediensozialisation}

Als Sozialisationsindikatoren werden das Geschlecht, das Alter und die Bildung in der Studie berücksichtigt. Die Fernsehsozialisation im Elternhaus wurde über den Grad der Zustimmung zu der Aussage „In meinem Elternhaus wurde viel ferngesehen“ auf einer 5er-Skala erhoben. ${ }^{20}$

\subsection{Unabbängige Variablen: Restriktionen und Optionen}

Folgende interne und externe Restriktionen und Optionen wurden in den Analysen berücksichtigt: 1) die Zahl der verfügbaren TV-Geräte im Haushalt (Durchschnitt: 1,8 Geräte); 2) die Zahl der Räume ohne TV (Durchschnitt: in 2 von 3 Räumen befindet sich kein TV); 3) beruflich bedingte Zeit außer Haus (Durchschnitt: 25 Stunden pro Woche); 4) Arbeitszeit zu Hause (Hausarbeit und berufliche Heimarbeit zusammen im Durchschnitt 15 Stunden pro Woche); 5) Haushaltsgröße (Einpersonenhaushalte $13 \%$ der Befragten, Zweipersonenhaushalte $37 \%$, größere Haushalte $49 \%$; 6) Leben in einer Partnerschaft: (81\% mit Partner, $19 \%$ ohne Partner); 7) subjektiv empfundene Erschöpfung (interne Restriktion) ${ }^{21}$ und 8) kognitive Kapazität (interne Restriktion) ${ }^{22}$.

\subsection{Unabhängige Variablen: Fernseh-Bindung}

Die Bindung an das Fernsehen wurde zum einen in Anlehnung an die Vermissensfrage (Ridder et al. 2002) operationalisiert und zum anderen wurde eine Frage gestellt, die stärker auf die situative Einbindung des Fernsehens abzielt ${ }^{23}$. Auch bei diesen Aussagen wurde die Zustimmung auf einer 5er-Skala gemessen.

19 Eigenwert 1,0; Items (Faktorladungen): Sportsendungen (.85), Krimis (.51).

$20 \mathrm{Im}$ Nachhinein erscheint diese Frage zur Mediensozialisation nur bedingt aussagekräftig, da fast die Hälfte der Befragten noch ohne Fernsehen aufgewachsen ist. Die Antworten korrelieren von daher deutlich mit dem Alter $(r=-.47)$ und die Zustimmung zu der Aussage ist insgesamt nur gering $(\varnothing=2,1)$.

21 Dazu wurde die Zustimmung zu der Aussage „Ich bin oft so müde, dass ich einfach nur noch fernsehen möchte" auf einer 5er-Skala (1-5) erhoben. Die Zustimmung zu der Aussage ist insgesamt nur gering $(\varnothing=2,2)$.

22 Dazu wurde die Zustimmung auf einer 5er-Skala (1-5) zu den folgenden Aussagen ermittelt: a) „Gleichzeitig fernzusehen und etwas anderes zu tun, finde ich anstrengend.“ und b) „Es stört mich, wenn ich etwas machen möchte und gleichzeitig der Fernseher läuft." Die beiden Variablen korrelieren stark miteinander $(r=.58)$ und konnten deshalb zu einem Index zusammengefasst werden. Die kognitive Überlastung wird von den Befragten im Durchschnitt als mittelstark erlebt $(\varnothing=3,1)$.

23 „Ein Leben ohne Fernsehen kann ich mir sehr gut vorstellen“. (Mittelwert gedreht: 3,3), „Wenn der Fernseher nicht läuft, fehlt mir etwas“ (1,7); Index: TV-Bindung (Korrelation zwischen den Items: $r=.32$, Mittelwert 2,5) 


\subsection{Unabbängige Variablen: Stimmungslagen}

Die Bedeutung der unterschiedlichen Stimmungslagen für die Befragten wurde durch insgesamt 16 Items operationalisiert. Mit jeweils vier Items sollte jede der vier Stimmungs-Hauptdimensionen, die sich aus der Kombination der Bewertungs- und der Erregungsdimension ergeben, erhoben werden. Die vorhergesagte Dimensionalität konnte durch die verwendeten Indikatoritems insgesamt recht gut abgebildet werden ${ }^{24}$. Allerdings mussten fünf der 16 Items aus dem Modell entfernt werden, da eine klare Zuordnung zu den Faktoren nicht möglich war. Insgesamt zeigt sich jedoch, dass das ursprünglich für die Erfassung situationsbezogener Befindlichkeiten und Stimmungen entwickelte Modell (Abele-Brehm und Brehm 1986) auch für die Erfassung langfristig vorherrschender Stimmungslagen geeignet ist.

Die Mittelwerte der vier Stimmungsdimensionen ${ }^{25}$ belegen, dass sich die Befragten überwiegend in einer positiv erregten Stimmungslage befinden (Energievoll-euphorisch $\varnothing=3,2)^{26}$. An zweiter Stelle folgt der Ärger $(\varnothing=2,8)^{27}$. D. h. auch dann, wenn sich die Personen in negativer Stimmung befinden, dominiert das hohe Erregungsniveau. Knapp dahinter folgt die Besinnlichkeit, bei der es sich um eine positive Stimmungslage auf niedrigem Erregungsniveau handelt $(\varnothing=2,7)^{28}$. Die depressive Stimmung - eine Kombination aus geringer Erregung und negativer Valenz - findet sich am seltensten $(\varnothing=2,5)^{29}$.

\subsection{Unabbängige Variablen: Kommunikationsbezogene Motive und wahrgenommene Leistungen}

Insgesamt vier kommunikationsbezogene Motivdimensionen werden im Rahmen der vorliegenden Untersuchung berücksichtigt. Zwei davon sind eher kognitionsbezogen, die anderen beiden eher affektiv-rekreationsbezogen (vgl. Rheinberg 2004: 12; Vowe \& Wolling 2004: 170 f.): Eher kognitionsbezogen sind das Informationsmotiv sowie das Organisationsmotiv, rekreationsbezogen sind das Motiv der Anregungssuche und das Escape-Motiv. Durch eine Faktorenanalyse konnte die Dimensionalität bestätigt werden, 60 \% der Varianz wurden durch vier Faktoren erklärt (vgl. Tabelle 3).

24 Hauptkomponentenanalyse mit Varimaxrotation; Vorgabe: 4 Faktoren-Lösung; 67 \% erklärte Varianz.

25 Die Einleitungssequenz zum Fragenblock lautet: „Wie häufig kommt es vor, dass Sie...“ Die Antworten wurden mit einer 5er-Skala von $1=$ „nie“ bis $5=$ „sehr oft“ erhoben.

26 Eigenwert 3,4; Items (Faktorladungen): ... das Gefühl haben, Sie könnten Bäume ausreißen? $(.82) ; \ldots$ sich tatkräftig und voller Energie fühlen? (.78); ... so richtig gut gelaunt sind? (.76); ... sich ganz unbeschwert fühlen? (.68). Alpha $=.78$.

27 Eigenwert 0,9; Items (Faktorladungen): ... gereizt sind? (.78), ... über etwas, was Sie erlebt haben, sehr wütend sind? (.78).

28 Eigenwert 1,1; Items (Faktorladungen): ...in einer verträumten Stimmung sind? (.88), ...ganz in Gedanken versunken sind? (.75).

29 Eigenwert 1,9; Items (Faktorladungen): ...sich sehr traurig fühlen? (.83); ...in einer gedrückten, niedergeschlagenen Stimmung sind? (.79); ...sich lustlos fühlen? (.69). Alpha = .75. 


\begin{tabular}{|c|c|c|c|c|}
\hline & 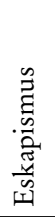 & 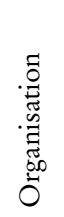 & 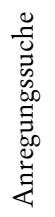 & 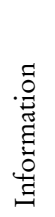 \\
\hline $\begin{array}{l}\text { Manchmal wünsche ich mir, all meine Probleme vergessen zu } \\
\text { können. }\end{array}$ & .85 & & & \\
\hline Ich habe oft den Wunsch, nicht so allein zu sein. & .74 & & & \\
\hline Ich wünsche mir oft, dem Alltag entfliehen zu können. & .71 & & .35 & \\
\hline $\begin{array}{l}\text { Es ist wichtig für mich, mein tägliches Leben optimal zu planen } \\
\text { und einzuteilen. }\end{array}$ & & .79 & & \\
\hline $\begin{array}{l}\text { Es ist mir wichtig, mich auf das, was ich tue, voll und ganz zu } \\
\text { konzentrieren. }\end{array}$ & & .75 & & \\
\hline Ich versuche, jede freie Minute sinnvoll zu nutzen. & & .74 & & \\
\hline $\begin{array}{l}\text { Es stört mich, wenn im Hintergrund ständig Geräusche, } \\
\text { Gespräche oder Musik zu hören sind. (gedreht) }\end{array}$ & & & .75 & \\
\hline $\begin{array}{l}\text { Wenn ich mich richtig konzentrieren will, brauche ich eine Art } \\
\text { Geräuschkulisse im Hintergrund. }\end{array}$ & & & .71 & \\
\hline Es ist für mich wichtig, Spannendes und Aufregendes zu erleben. & & & .63 & \\
\hline $\begin{array}{l}\text { Es ist mir wichtig, über aktuelle Ereignisse umfassend informiert } \\
\text { zu werden. }\end{array}$ & & & & .80 \\
\hline $\begin{array}{l}\text { Von wichtigen Ereignissen in der Welt möchte ich möglichst } \\
\text { schnell erfahren. }\end{array}$ & & & & .77 \\
\hline $\begin{array}{l}\text { Es ist mir wichtig, im Bekanntenkreis auf vielen Gebieten } \\
\text { mitreden zu können. }\end{array}$ & & & & .66 \\
\hline Eigenwerte & 2,0 & 2,4 & 1,2 & 1,6 \\
\hline Indexmittelwert & 2,8 & 3,7 & 2,6 & 4,0 \\
\hline Alpha & .67 & .67 & .52 & .64 \\
\hline
\end{tabular}

Hauptkomponentenanalyse mit Varimaxrotation; 60 \% erklärte Varianz; alle Faktorladungen > .30

Neben den vier kommunikationsbezogenen Motiven wurden auch die wahrgenommenen Leistungen (Gratifications obtained des GS/GO-Modells) erhoben. Sie können sich zum einen auf das Fernsehen allgemein beziehen und zum anderen auf die situationsbezogenen Effekte der Nebenbeinutzung. Besondere Erklärungskraft für die Unterschiede in der Nebenbeinutzung haben vermutlich die speziell auf den Modus der Nebenbeinutzung abzielenden Leistungsbewertungen, auch wenn über alle Befragten hinweg dieser Leistungsdimension des Fernsehens im Vergleich zu den anderen vier Dimensionen keine besondere Bedeutung zugeschrieben wird (Tabelle 4). 
Tabelle 4: Leistungen der Nebenbeinutzung des Fernsehens (Skalenmittelwerte*)

Wenn der Fernseher im Hintergrund läuft, schafft das eine angenehme Atmosphäre. $\quad 2,1$

Wenn nebenbei der Fernseher läuft, geht mir vieles leichter von der Hand. $\quad 1,9$

Wenn der Fernseher im Hintergrund läuft, hilft das, Gesprächspausen zu überbrücken. $\quad 1,9$

Indexmittelwert (Alpha $=.81)$

* Ser-Skala von 1 "stimme gar nicht $z u$ " bis 5 "stimme voll $z u$ “

Bei den auf die generelle Fernsehnutzung bezogenen Leistungseinschätzungen wurden zwei Dimensionen unterschieden: Zum einen wird auch hier wiederum das Escape-Konzept berücksichtigt und zum anderen wird die wahrgenommene Leistung des Fernsehens als Zeitmanager untersucht. Die beiden Dimensionen konnten durch eine Faktoranalyse bestätigt werden (Tabelle 5).

Tabelle 5: Leistungen des Fernsehens

\begin{tabular}{lcc}
\hline & $\begin{array}{c}\text { TV } \\
\text { ist } \\
\text { Zeitmanager }\end{array}$ & $\begin{array}{c}\text { TV } \\
\text { ermöglicht } \\
\text { Escape }\end{array}$ \\
\hline Manchmal schaue ich Fernsehen nur um die Zeit herumzu- & .89 & \\
bringen. & & \\
Um Zeit zu überbrücken, schalte ich oft den Fernseher ein. & .87 & \\
Das Fernsehen hilft mir den Tag einzuteilen. & .55 & .86 \\
\hline Das Fernsehen hilft manchmal, die Sorgen und Probleme des & & .81 \\
Alltags zu vergessen. & & .72 \\
Das Fernsehen sorgt für Ablenkung. & .38 & 1,3 \\
Fernsehen beruhigt mich, wenn ich Ärger habe. & 2,8 & 2,7 \\
\hline Eigenwerte & 2,0 & .71 \\
Indexmittelwerte & .76 & \\
Alpha & & \\
\hline
\end{tabular}

Hauptkomponentenanalyse mit Varimaxrotation; 67 \% erklärte Varianz; alle Faktorladungen $>.30$

Die Leistung des Zeitmanagements thematisiert - ähnlich wie das Organisationsmotiv - die Relation zwischen Fernsehnutzung und Zeitverwendung.

\subsection{Unabbängige Variablen: Qualitätswabrnehmung}

Um zu überprüfen, ob die wahrgenommene Qualität des Fernsehens und des Radios einen Einfluss auf die Nebenbeinutzung haben, wurde für beide Medien die Einschätzung der derzeitigen Qualität erfragt. Weiterhin wurde ermittelt, ob die Befragten eine Veränderung des Angebots zum Besseren oder zum Schlechteren wahrgenommen haben. Auch hier wurde wiederum die Zustimmung zu den Items auf 5er-Skalen erhoben. Die Ergebnisse verdeutlichen, dass die Leistung beider Medien eher positiv als negativ beurteilt wird. Beim Fernsehen wird jedoch eine gewisse Tendenz zum Negativen, beim Radio hingegen eine leichte Verbesserung der Angebotsqualität konstatiert (Tabelle 6). 


\section{Tabelle 6: Qualitätswabrnehmung des Fernsehens und des Radios (Mittelwerte*)}

Das Fernsehen bringt die neuesten Nachrichten besonders schnell. $\quad 4,0$

Das Fernsehen bringt die Dinge fesselnd und interessant. $\quad 3,5$

Im Fernsehen kommt nur selten etwas, wo es sich lohnt, aufmerksam zuzuschauen $\quad 3,2$ (gedreht).

Im Fernsehen kommen oft spannende und interessante Sendungen. 3,1

Im Fernsehen läuft irgendwo immer etwas Interessantes. $\quad 2,8$

Das Fernsehen zeigt die Dinge so, wie sie wirklich sind. $\quad 2,5$

Index: Qualitätswahrnehmung des Fernsehens (Alpha = .78) 3,2

Das Fernsehprogramm ist in den letzten Jahren immer besser geworden. 2,5

Das Fernsehprogramm ist in den letzten Jahren immer schlechter geworden (ge- $\quad 3,0$ dreht).

Index: Wabrnehmung Qualitätsveränderung des Fernsehens $(r=.70) \quad 2,8$

Das Radio bringt die neuesten Nachrichten besonders schnell. 3,9

Das Radio informiert über alles Wichtige, was geschieht. $\quad 3,8$

Die Musik im Radio gefällt mir nicht (gedreht). 3,7

Im Radio kommt nur selten etwas, wo es sich lohnt, aufmerksam zuzuhören (ge- $\quad 3,4$ dreht).

Index: Qualitätswabrnehmung des Radios (Alpha=.71) 3,6

Das Radioprogramm ist in den letzten Jahren immer besser geworden. $\quad 3,3$

Das Radioprogramm ist in den letzten Jahren immer schlechter geworden (gedreht). 3,7

Index: Wabrnehmung Qualitätsveränderung des Radios $(r=.63) \quad 3,5$

*Ser-Skala von 1 „stimme gar nicht $\mathrm{zu}$ “ bis 5 „stimme voll $\mathrm{zu}$ “

\section{Analytische Ergebnisse}

Zunächst wird nun überprüft, ob die oben erläuterten Faktoren einen unmittelbaren Einfluss auf die Nebenbeinutzung haben. Als abhängige Variable wurden neben dem generellen Umfang der Nebenbeinutzung auch die audioorientierte und die sozialorientierte Nebenbeinutzung untersucht. Weiterhin haben wir die beiden tätigkeitsbezogenen Formen der Nebenbeinutzung (Pflicht- und Freizeittätigkeiten) sowie die vier inhaltsbezogenen Indizes (Unterhaltung und Infotainment im Nachmittagsprogramm, Information, Musik \& Comedy, Krimi \& Sport) berücksichtigt. Auf diese neun Indikatoren der Fernsehnebenbeinutzung wurden mit den zuvor vorgestellten 29 unabhängigen Faktoren (Variablen und Indizes) Regressionen berechnet. ${ }^{30}$ Der Einfluss der verschiedenen Einflussfaktoren wurde in einem mehrstufigen Verfahren bestimmt. ${ }^{31}$ Die

30 Auf die vier inhaltsbezogenen Indikatoren der Nebenbeinutzung wurden zunächst Regressionsmodelle gerechnet mit der generellen Nutzung des jeweiligen Genres als unabhängiger Variable. Die Residuen dieser Regressionen bilden in den nachfolgenden Analysen die abhängige Variable. Auf diese Weise wird sichergestellt, dass die Nebenbeinutzung bestimmter Inhalte nicht mit den allgemeinen Genrepräferenzen konfundiert wird.

31 Zunächst wurden mit der Forward- und der Backward-Option alle Variablen ermittelt, die einen signifikanten Effekt haben. Dann wurde geprüft, ob sich diese Variablen auch in einem einfachen Modell (Enter-Option) als erklärungskräftig erweisen. Nur Variablen, die auch hier einen signifikanten Effekt hatten, wurden im Modell belassen. Zugleich wurde geprüft, ob zwischen den unabhängigen Variablen Multikollinearitätsprobleme bestehen. In einigen Fällen sind 
Ergebnisse sind in Tabelle 7 dargestellt. Nachdem die direkten Effekte ermittelt worden waren, haben wir als nächstes geprüft, ob sich möglicherweise indirekte Effekte nachweisen lassen, wie sie durch das Modell in Grafik 1 nahegelegt werden. Dies wurde am Beispiel der Nebenbeinutzung von Unterhaltungsangeboten realisiert. Die unabhängigen Variablen, die sich in diesem Modell als erklärungskräftig erwiesen haben, sind in weiteren Regressionen als abhängige Variable verwendet worden. Bei diesen Faktoren wurde also eine Pfadanalyse durchgeführt. Die Ergebnisse dieser Regression werden in Tabelle 8 dargestellt. In beiden Tabellen sind nur die signifikanten $(\mathrm{p}<.05)$ beta-Koeffizienten ausgewiesen.

\subsection{Generelle Effekte}

Drei Faktoren erweisen sich nicht nur in der Mehrheit der Modelle als erklärungskräftig, sondern haben zudem auch meistens einen besonders starken Einfluss: Dabei handelt es sich um die subjektive Restriktion der kognitiven Überlastung, die wahrgenommene Leistung der Fernsebnebenbeinutzung sowie die Wabrnebmung der Fernseb-Programmqualität.

Personen, die sich durch die Nebenbeinutzung kognitiv überlastet fühlen, schauen weniger nebenbei fern. Dieser Effekt war zu erwarten, da kaum anzunehmen ist, dass Menschen längere Zeit mit einem unangenehm störenden Medium verbringen, wenn sich dieses auch mit einem Knopfdruck ausschalten lässt. Trivial ist der Befund trotzdem nicht, zeigt sich doch, dass sich die kognitive Überlastung weder auf die sozialorientierte Nebenbeinutzung noch auf die Fernsehnutzung bei Erledigung von Pflichtaufgaben auswirkt. Pflichtaufgaben können in der Regel so routiniert erledigt werden, dass eine Überlastung kaum auftritt. Auch bei den übrigen abhängigen Variablen variiert die Stärke des Einflusses der kognitiven Überlastung: Die audioorientierte Nebenbeinutzung sowie spannende TV-Sendungen im Hintergrund werden demnach als besonders kognitiv belastend empfunden - hier ist wohl der Ablenkungseffekt und damit die Konzentrationsstörung bei der Paralleltätigkeit am größten. Dass auch die Nebenbeinutzung von Comedy und Musik sowie die Rezeption des unterhaltungs- und infotainmentorientierten Nachmittagsprogramms nicht durch kognitive Überlastung behindert werden, stützt diese Interpretation.

Weiterhin sehen Personen, die die Situation der Fernsehnebenbeinutzung als positiv wahrnehmen (vgl. Tab. 7: „Leistung der Nebenbeinutzung“), häufiger nebenbei fern. Die Wahrnehmung der Nebenbeinutzungssituation als angenehm wirkt sich auf fast alle Formen und Inhalte der Nebenbeinutzung stark aus, nur auf die Rezeption von Informationsangeboten sowie von Krimis und Sport ist der Effekt deutlich schwächer. Es erscheint äußerst plausibel, dass informierende Angebote nicht deswegen nebenbei genutzt werden, weil die Situation durch den laufenden Fernseher positiv gestaltet wird, sondern weil ein besonders starkes Informationsmotiv vorhanden ist. Tatsächlich ist festzustellen, dass dieses Motiv sowohl die Nebenbeinutzung von Informationssendungen als auch die von Krimis und Sport positiv beeinflusst. Vermutlich steht bei diesen beiden Angebotssegmenten der Inhalt des Programms noch etwas stärker im Mittelpunkt der Aufmerksamkeit und die anderen Dinge werden nebenher erledigt, während

problematische Variablen entfernt worden. Abschließend wurde dann bei allen unabhängigen Variablen noch einmal einzeln geprüft, ob sie in dem nun bereits weitgehend fertig gestellten Modell noch zusätzliche Varianz erklären können. 
Tabelle 7: Erklärungsmodelle: Direkte Einflüsse (Regressionen)

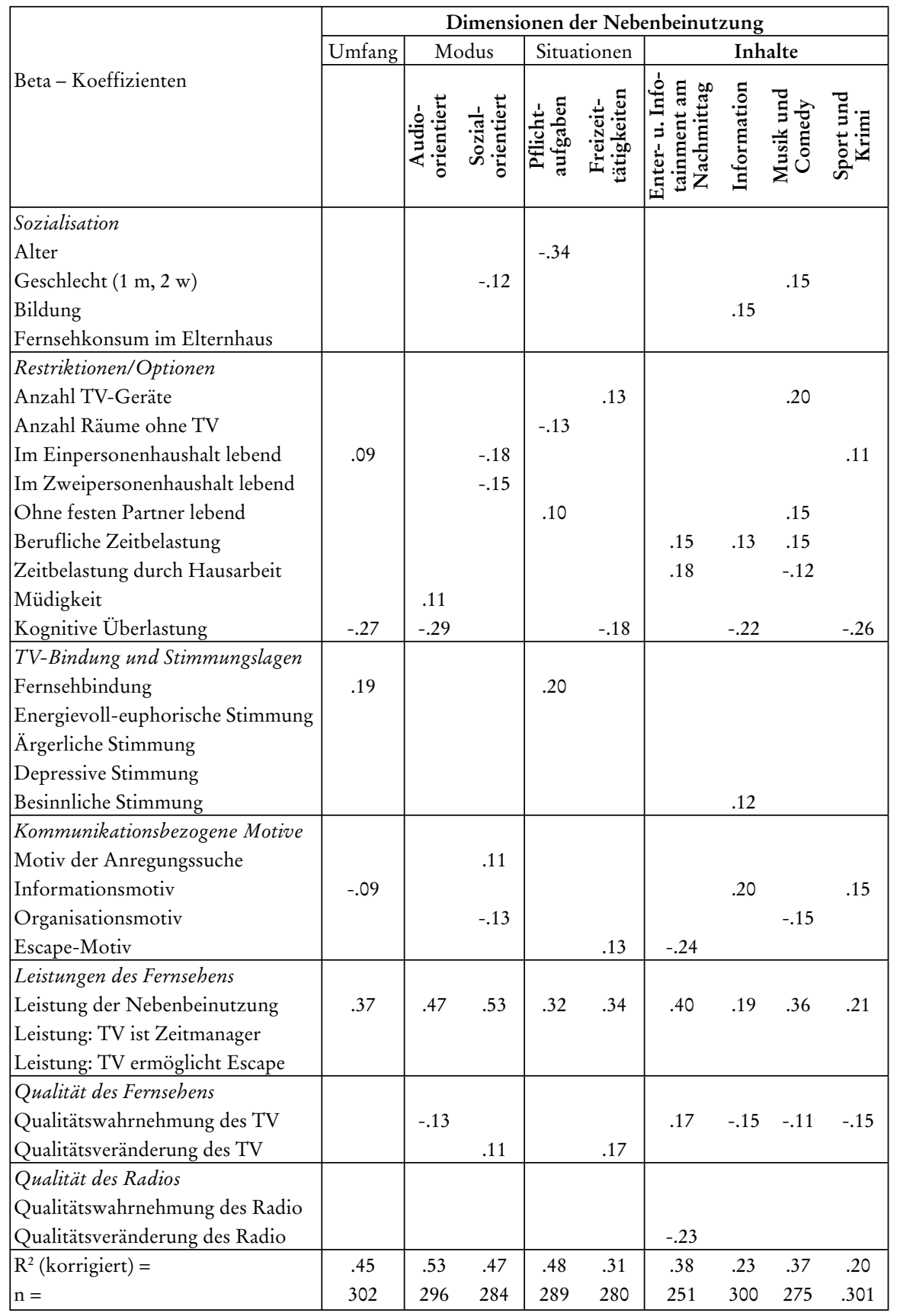


bei den anderen untersuchten TV-Angeboten sich die Relation umgekehrt hat: Hier ist die spezifische Leistung als Hintergrundmedium ausschlaggebend für die Nutzung.

Eine geringere Effektstärke weist die Wahrnehmung der Qualität bzw. der Veränderung der Qualität des Fernsehprogramms auf. Bezüglich der Qualität hielten wir Effekte in beide Richtungen für plausibel: Ein gutes Fernsehprogramm wird unter Umständen besonders aufmerksam und damit seltener nebenbei genutzt. Womöglich ist ein gutes Programm für die Rezipienten aber auch so attraktiv, dass man selbst dann nichts verpassen will, wenn primär andere Tätigkeiten ausgeübt werden. Dies würde dann zu einer häufigeren Nebenbeinutzung führen. Die erste Annahme dominiert in den Befunden: Nur beim nachmittäglichen Unterhaltungs- und Infotainmentprogramm wirkt sich eine positive Qualitätswahrnehmung positiv auf die Nebenbeinutzung aus. Dies ist verständlich, da die zu dieser Tageszeit anliegenden Arbeiten eine aufmerksame Nutzung häufig ausschließen werden. Bei Informationssendungen, Comedy und Musik sowie bei Krimis und Sport, aber auch beim Modus des „Fernhörens“ ist der Zusammenhang dagegen negativ. Hier führt ein positives Qualitätsurteil zu einer vorwiegend aufmerksamen Fernsehnutzung ${ }^{32}$. Andere Effekte hat hingegen die wahrgenommene Veränderung der Programmqualität: Wer das Fernsehprogramm als verbessert erlebt, lässt den Fernseher in Interaktionssituationen und bei Freizeittätigkeiten häufiger nebenbei laufen.

\subsection{Differentielle Effekte}

Neben den zwei beschriebenen generellen Effekten finden sich zahlreiche Einflussfaktoren, die nur bei einzelnen abhängigen Variablen Effekte zeigen.

\section{Umfang der Nebenbeinutzung}

Neben den generellen Einflussgrößen hat besonders die Bindung an das Medium Fernsehen einen positiven Einfluss auf den zeitlichen Umfang der Nebenbeinutzung. Auf das Fernsehen nicht verzichten zu können, heißt also auch, es nicht missen zu wollen, wenn andere Tätigkeiten anstehen. Daneben gibt es nur noch zwei weitere, allerdings schwache Einflüsse: Negativ wirkt sich eine positive Ausprägung des Informationsmotivs aus: Wer im Fernsehen Informationen sucht, gehört zu denen, die eher weniger nebenbei fernsehen. Häufiger nutzen dagegen Personen das Fernsehen nebenbei, die in Einpersonenhaushalten leben. Singles bietet sich demnach häufiger die Gelegenheit, den Fernseher nebenbei laufen zu lassen, während dies in größeren Haushalten offenbar nicht so oft möglich ist.

\section{Audioorientierte Nebenbeinutzung}

„Fernhören“ ist diejenige Form der Nebenbeinutzung, die insgesamt am besten durch die unabhängigen Variablen erklärt werden kann. Neben den beschriebenen generellen Effekten findet sich allerdings nur noch ein weiterer Einflussfaktor. Einen schwachen Einfluss hat die Müdigkeit: Wer oft so müde ist, dass er nichts anderes mehr machen möchte als fernzusehen, der lässt häufiger den Fernseher als Geräuschkulisse laufen.

32 Eine entsprechende Analyse belegt dies: Das Qualitätsurteil korreliert mit der Gesamtnutzung aller Inhaltsgruppen signifikant positiv. 


\section{Sozialorientierte Nebenbeinutzung}

Das Ausmaß der sozialorientierten Nebenbeinutzung ist hingegen stärker durch die Sozialisation und die objektiven Restriktionen bestimmt. Den stärksten Einfluss hat die Haushaltsgröße: In größeren Haushalten ist diese Nutzungsform deutlich häufiger zu finden, was zeigt, dass die sozialen Interaktionspartner beim Fernsehen wohl meist Familienmitglieder sind.

Positiv wirkt ferner das Motiv der Anregungssuche: Denkbar ist, dass das im Hintergrund laufende Fernsehprogramm Themen für die Gespräche liefert. Das Organisationsmotiv hält die Befragten dagegen eher von dieser Form der Nebenbeinutzung ab: Für das Bedürfnis, die Zeit gut zu verwenden, ist die Trennung von sozialer Interaktion und Mediennutzung erforderlich. Nicht ganz so leicht zu interpretieren ist schließlich der Einfluss des Geschlechts: Männer schauen, während sie mit anderen sozial interagieren, häufiger fern als Frauen. Möglicherweise ist dies Folge davon, dass Männer dem sozialen Austausch vielfach eine etwas geringe Wichtigkeit zuweisen als Frauen.

\section{Situation: Pflichtaufgaben}

Die Erklärung des Nebenbeisehens bei Pflichtaufgaben zeigt den stärksten und wohl auch interessantesten differentiellen Effekt: Das Alter hat hier einen sehr starken negativen Einfluss (beta -.34). Arbeit und Fernsehen kombinieren vor allem die jüngeren Befragten, während ältere Befragte dies eher trennen. Dies lässt sich nur zum Teil durch die größere Fähigkeit zur Parallelverarbeitung bei Jüngeren erklären - was die später präsentierte Pfadanalyse belegt. Die entsprechende Variable „kognitive Überlastung“ zeigt hier nicht den bekannten negativen Einfluss, sondern wird durch die Variable Alter verdrängt. Die Annahme liegt nahe, dass hier normative Vorstellungen in Bezug auf Arbeit eine Rolle spielen, die zwischen „preußischen Tugenden“ und hedonistischer Spaßgesellschaft variieren.

Deutlich schwächer sind die übrigen Einflüsse: Die Zahl der Räume ohne Fernsehgeräte wirkt sich negativ aus. Dies ist unmittelbar plausibel, sind doch viele Arbeiten im Haushalt an andere Räume als das Fernsehzimmer gebunden. Einen positiven Einfluss hat die Fernsehbindung: Wenn jemand ohne Fernsehen nicht leben zu können meint, dann wirkt sich dies auch auf die Integration der Fernsehnutzung als Paralleltätigkeit bei der Arbeit aus. Ferner sehen Singles häufiger parallel zu Pflichtaufgaben fern.

\section{Situation: Freizeittätigkeiten}

Da viele Freizeitaktivitäten an bestimmte Räume der Wohnung gebunden sind, ist der positive Einfluss der Geräteausstattung plausibel: Je mehr Geräte sich im Haushalt befinden, desto häufiger begleitet das Fernsehprogramm die Freizeit. Doch warum sollte jemand, der sich zum Beispiel entspannenden Hobbys widmet, überhaupt nebenbei fernsehen oder - hören? Hier sind zusätzliche Motive zu erwarten, und so überrascht es nicht, dass hier das Eskapismusmotiv einen - wenn auch schwachen - positiven Einfluss hat - im Gegensatz zu allen anderen Modellen, in denen es keine derartige Rolle spielt. 


\section{Inhalt: Unterhaltung und Infotainment am Nachmittag}

Die Nebenbeinutzung leichter Unterhaltungs- und Infotainmentangebote hängt von einer ganzen Reihe von Faktoren ab. Da die hier abgebildeten Fernsehformate vor allem nachmittags ausgestrahlt werden, erklärt sich der positive Einfluss der Zeitbelastung durch Hausarbeit sowie der allgemeinen beruflichen Belastung. Hier wird offensichtlich das Radio durch das Fernsehen als Begleitmedium abgelöst: Die Wahrnehmung einer verschlechterten Radioqualität wirkt sich genauso positiv auf den Nebenbeikonsum solcher Sendungen aus wie die bereits oben (Kapitel 4.1) diskutierte positive Beurteilung der TV-Programmqualität.

Der negative Einfluss des Eskapismus-Motivs überrascht und lässt sich am ehesten mit einer umgekehrten Kausalität erklären: Wer durch die Begleitmediennutzung seine Arbeit angenehmer gestaltet, verringert damit sein Fluchtbedürfnis.

\section{Inhalt: Informationsangebote}

Interessanter Weise finden wir positive Effekte des Informationsmotivs und der Bildung auf die Nebenbeinutzung von Informationsangeboten. Nicht nur die aufmerksame Nutzung, sondern auch die Nebenbeinutzung wird also durch diese Faktoren beeinflusst. Der positive Einfluss besinnlicher Stimmungen auf die Nebenbeinutzung von Informationsangeboten könnte darauf zurückzuführen sein, dass Personen, bei denen diese Stimmungslagen vorherrschen, am wenigsten Mood Management durch die Geräuschkulisse benötigen, es aber andererseits als ganz angenehm empfinden, sich durch anspruchsvollere Inhalte nebenbei anzuregen. Eine starke berufliche Zeitbelastung wirkt sich ebenfalls positiv aus: Hier führt das Zeitmanagement wohl dazu, dass die Informationsaufnahme parallel zu anderen Tätigkeiten realisiert wird.

\section{Inhalt: Unterhaltungsfragmente Musik und Comedy}

Den stärksten zusätzlichen Einfluss auf die Nebenbeinutzung von Musik und Comedy hat hier die Anzahl der TV-Geräte im Haushalt. Insbesondere Musik kann in allen Räumen der Wohnung gut nebenbei genutzt werden, was sich aber nur bei entsprechender Ausstattung realisieren lässt. Frauen und Personen, die ohne festen Partner leben, lassen diese Inhalte - von denen eine stimmungsaufhellende Wirkung erwartet werden kann - besonders häufig im Hintergrund laufen. Negativ wirkt sich dagegen das Organisationsmotiv aus: Das Bedürfnis nach sinnvoller Zeitplanung führt eher zur Vermeidung solcher Geräuschkulissen. In diesem Zusammenhang sind auch die Zusammenhänge mit dem Zeithaushalt interessant: Eine starke berufliche Belastung befördert den Hintergrundkonsum dieser Angebote, viel Hausarbeit hemmt ihn dagegen. Hinter diesen Effekten verbirgt sich vermutlich ein komplexes Wechselspiel von Optionen und Restriktionen, die mit den unterschiedlichen kognitiven Anforderungen von Beruf und Hausarbeit zusammenhängen: Während in den meisten Fällen bei der Berufsarbeit allenfalls noch Musik im Hintergrund laufen kann, können die meisten Hausarbeiten auch durchaus durch Programme begleitet werden, die höhere Anforderungen an die Aufmerksamkeit stellen. 


\section{Inhalt: Spannung in Krimis und Sportsendungen}

Dass sich das Informationsmotiv auch auf die Nebenbeinutzung spannender TV-Inhalte positiv auswirkt, deutet darauf hin, dass diesen Sendungen auch bei geringer Aufmerksamkeit Informationen entnommen werden können: Dies sind zum einen sicherlich die Sportergebnisse und zum anderen wohl auch Eindrücke von der Polizeiarbeit, der Gerichtsmedizin sowie aktueller Probleme mit organisierter Kriminalität. Daneben hat nur noch das Leben in einem Einpersonenhaushalt einen schwachen positiven Einfluss auf die Nutzung dieser Inhalte.

\subsection{Indirekte Effekte}

Das oben vorgeschlagene Modell (Grafik 1) postuliert nicht nur direkte Effekte auf die Nebenbeinutzung, sondern auch indirekte Effekte (gestrichelte Linien im Modell). Um zu überprüfen, ob sich solche Effekte nachweisen lassen, sind Pfadanalysen durchgeführt worden. $\mathrm{Da}$ es im Rahmen des Beitrags nicht möglich ist, für alle neun abhängigen Variablen die Pfadmodelle vorzustellen, soll an dieser Stelle beispielhaft das Erklärungsmodell für die Nebenbeinutzung von Unterhaltung und Infotainment im Nachmittagsprogramm berechnet und erläutert werden ${ }^{33}$. Für die anderen Indikatoren der Nebenbeinutzung würden sich die Pfadmodelle aus anderen Faktoren zusammensetzen, je nachdem welche Variablen dort direkte Effekte gezeigt haben.

Es wurden Regressionen auf vier der vormals unabhängigen Variablen berechnet, die sich in dem zuvor vorgestellten Modell als bedeutsame Erklärungsfaktoren erwiesen hatten (Tabelle 8). Die Ergebnisse belegen, dass zahlreiche indirekte Effekte nachzuweisen sind. Einzelne Erklärungsfaktoren - insbesondere die Leistungswahrnehmungen der Nebenbeinutzung und die Qualitätswahrnehmung des TV - lassen sich in erheblichem Umfang durch andere im Modell integrierte Faktoren erklären. Wenn man sich anschaut, welche Faktoren hier als Erklärungsvariablen eine Rolle spielen, dann sind mehrere Befunde hervorzuheben: Zum einen zeigt sich, dass das Alter zwar keine direkten Effekte auf die Nebenbeinutzung von Unterhaltung hat, sich aber vielfach indirekt auswirkt. Drei der vier Erklärungsvariablen werden zum Teil ganz erheblich durch das Alter beeinflusst. Auch die Müdigkeit und die Bindung an das TV - die beide keinen direkten Einfluss ausüben - wirken sich indirekt aus, indem sie andere Faktoren beeinflussen, die ihrerseits direkte Effekte bei der Nebenbeinutzung hervorrufen. Bemerkenswert ist auch, wie sich die Stimmungslagen auf das Escapemotiv auswirken: Negative und dabei vor allem depressive Stimmungen fördern das Fluchtbedürfnis. Ähnliches gilt auch für die Motive und Leistungsbewertungen: Die Qualitätswahrnehmungen des Fernsehens werden - wie durch das Modell postuliert - durch diese Faktoren beeinflusst.

33 Diese Form der Nebenbeinutzung wurde ausgewählt, weil hier ein Modell mittlerer Komplexität entsteht, das gerade noch visualisierbar ist. 
Tabelle 8: Erklärungsmodelle: Indirekte Einflüsse (Regressionen)

\begin{tabular}{lllll}
\hline $\begin{array}{l}\text { Beta- } \\
\text { Koeffizienten }\end{array}$ & Escapemotiv & $\begin{array}{l}\text { Leistung der } \\
\text { Nebenbei- } \\
\text { nutzung }\end{array}$ & $\begin{array}{l}\text { Qualitäts- } \\
\text { wahrnehmung } \\
\text { TV }\end{array}$ & $\begin{array}{l}\text { Qualitätsver- } \\
\text { änderung } \\
\text { Radio }\end{array}$ \\
\hline $\begin{array}{l}\text { Sozialisation } \\
\text { Alter }\end{array}$ & -.17 & -.23 & .33 & \\
$\begin{array}{l}\text { Geschlecht } \\
\text { Bildung }\end{array}$ & -.13 & -.14 & -.11 & .14 \\
Mediensozialisation & & & & \\
\hline
\end{tabular}

Restriktionen/Optionen

Müdigkeit

Kognitive Überlastung

$\begin{array}{ll}-.31 & .19\end{array}$

Ohne festen Partner lebend .16

$\mathrm{x}$

$\mathrm{X}$

$\mathrm{X}$

TV-Bindung und

Stimmungslagen

Fernsehbindung

Stimmung

energievoll-euphorisch

Stimmung ärgerlich

Stimmung depressiv

.36

Stimmung besinnlich $-.09$

\begin{tabular}{|c|c|c|c|c|}
\hline \multicolumn{5}{|l|}{$\begin{array}{l}\text { Kommunikationsbezogene } \\
\text { Motive }\end{array}$} \\
\hline Informationsmotiv & $\mathrm{x}$ & & .20 & \\
\hline Organisationsmotiv & $\mathrm{x}$ & & & .14 \\
\hline \multicolumn{5}{|l|}{ Leistungen des Fernsehens } \\
\hline $\begin{array}{l}\text { Leistung der } \\
\text { Nebenbeinutzung }\end{array}$ & $\mathrm{x}$ & $\mathrm{x}$ & & \\
\hline $\begin{array}{l}\text { Leistung: TV ist } \\
\text { Zeitmanager }\end{array}$ & $\mathrm{x}$ & $\mathrm{x}$ & & -.25 \\
\hline $\begin{array}{l}\text { Leistung: TV ermöglicht } \\
\text { Escape }\end{array}$ & $\mathrm{x}$ & $\mathrm{x}$ & .31 & .18 \\
\hline $\mathrm{R}^{2}$ (korrigiert) $=$ & .36 & .58 & .47 & .19 \\
\hline $\mathrm{n}=$ & 281 & 281 & 281 & 263 \\
\hline
\end{tabular}

Bei den mit x gekennzeichneten Feldern wurden die jeweiligen Variablen bei den Regressionen nicht berücksichtigt, da durch das Modell keine Zusammenhänge postuliert wurden. 
Grafik 2: Einflussfaktoren auf die Nebenbeinutzung von Unterhaltungs-und Infotainmentangeboten im Nachmittagsprogramm

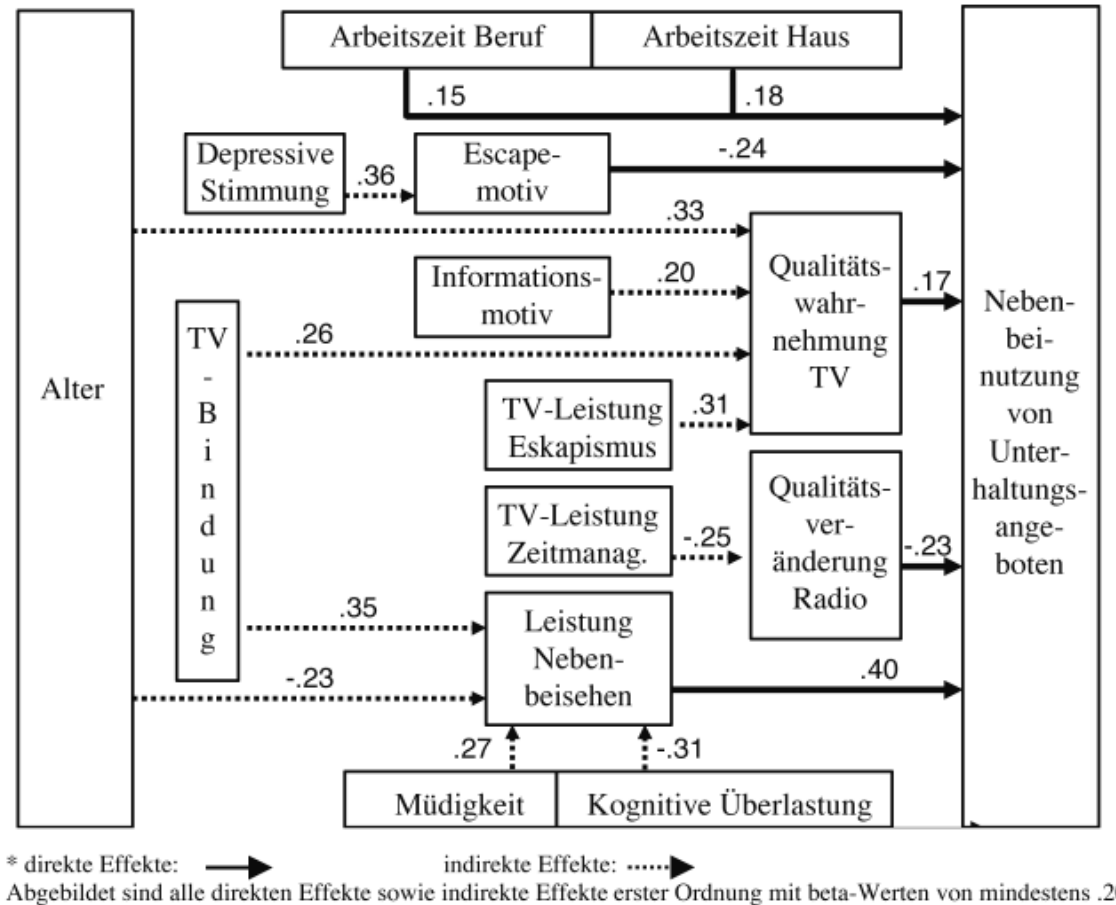

Es ist an dieser Stelle nicht möglich, die zahlreichen indirekten Effekte ausführlich zu würdigen. Es kann hier nur beispielhaft erläutert werden, wie sie zu interpretieren sind. Der Grafik 2 ist zu entnehmen, dass die Qualitätswahrnehmung einen positiven Effekt auf die Nebenbeinutzung von Unterhaltungsangeboten hat. Die Qualitätswahrnehmung des Fernsehprogramms wird aber wiederum durch die Escape-Leistungswahrnehmung beeinflusst. Wer also dem Fernsehen stärker zutraut, dass es ihm zur Flucht aus der Realität verhelfen kann, beurteilt die Qualität des Programms positiver. Die wahrgenommene Leistung des TV als Mittel zur Realitätsflucht wirkt sich somit indirekt auf die häufigere Nebenbeinutzung von unterhaltenden TV-Angeboten aus. Die Stärke des indirekten Effekts lässt sich abschätzen, indem man die beta-Koeffizienten miteinander multipliziert $(0.31 * 0.17=0.05)$. Da auch das Alter und die Fernsehbindung ähnliche Effekte auf die Qualitätswahrnehmung des TV haben, wirken sich auch diese Faktoren in indirekter Weise aus. Alter und Fernsehbindung beeinflussen jedoch zusätzlich auch die Leistungswabrnebmung der Nebenbeinutzung, die wiederum einen starken Effekt auf die Nebenbeinutzung von Unterhaltung hat. Das Alter und die Fernsehbindung haben damit nicht nur via Qualitätswahrnehmung, sondern auch vermittelt über die wahrgenommene Leistung der Nebenbeinutzung einen Einfluss auf die Häufigkeit der Nebenbeinutzung von Unterhaltung. 


\section{Resümee}

Die Nebenbeinutzung des Fernsehens ist nach den vorliegenden Befunden vor allem ein Mittel, das Menschen nutzen, um Situationen in ihrem Alltag zu gestalten. Dafür spricht, dass sich jene Faktoren, die sich auf das situationsbezogene Erleben der Nebenbeinutzung beziehen (kognitive Überlastung und Leistung der Nebenbeinutzung), durchgängig als einflussreich erweisen. Dafür spricht auch, dass sich die häuslichen Rahmenbedingungen (Haushaltsgröße, Geräteausstattung) auf das Ob und das Wie der Nebenbeinutzung auswirken. Das Fernsehen ist aber dennoch - auch bei der Parallelnutzung - mehr als nur eine reine Geräuschkulisse, denn die situativen Rahmenbedingungen, die Motive und die Qualitätswahrnehmungen wirken sich auf die Nebenbeinutzung der unterschiedlichen Inhalte verschieden aus. Solche differenzierten Effekte lassen sich nur dadurch erklären, dass die Fernsehinhalte auch im Modus der Nebenbeinutzung wahrgenommen werden und für die Nutzungsentscheidungen bedeutsam sind.

Die Fernsehnutzung hat sich in den letzten Jahren und Jahrzehnten stark verändert und wird sich in Zukunft weiter verändern. Ob die hier untersuchten Modi der Nebenbeinutzung an Bedeutung gewinnen werden oder ob sich möglicherweise völlig andere Formen der TV-Nutzung entwickeln werden, ist schwer abzuschätzen. Es spricht aber einiges dafür, dass die Nebenbeinutzung generell eine wesentliche Form der Nutzung bleiben wird. Unsere Ergebnisse haben gezeigt, dass sich die Geräteausstattung auf die Art der TV-Nutzung auswirkt. Neue Technologien (Festplattenrecorder, EPGs) und mobile Abspielgeräte (Handys, Laptops) werden den Rezipienten neue Möglichkeiten der (Nebenbei-)Nutzung eröffnen.

Die Befunde verdeutlichen aber ebenfalls, dass auch die sozialen Rahmenbedingungen die Nebenbeinutzung beeinflussen. Von daher ist damit zu rechnen, dass auch die sich ändernden Lebensgewohnheiten (beispielsweise die zunehmende Anzahl von Alleinlebenden) Auswirkungen auf die Fernsehnutzung haben werden. Es wird Aufgabe der Kommunikationswissenschaft sein, die technischen und gesellschaftlichen Veränderungen genau zu beobachten, um so frühzeitig auf Veränderungen im Umgang mit dem Medienangebot aufmerksam zu werden. Eine theoretisch fundierte Kommunikationswissenschaft darf jedoch nicht bei der möglichst frühzeitigen Registrierung von Entwicklungen stehen bleiben, sondern muss versuchen, Modelle zu entwickeln, mit denen die Nutzungsentscheidungen der Rezipienten erklärt werden können. Ein solches Modell wurde hier vorgestellt und hinsichtlich seiner Erklärungskraft geprüft. Dabei hat sich der Anschluss an etablierte theoretische Ansätze der Kommunikationswissenschaft als äußerst fruchtbar erwiesen. Allerdings war es notwendig, diese Ansätze weiterzuentwickeln. $\mathrm{Zu}$ nennen sind hier insbesondere die kognitiven Restriktionen sowie die spezifischen Leistungen der Nebenbeinutzung, die sich in vielen Modellen als besonders einflussreich herausgestellt haben. Vor allem hat sich jedoch gezeigt, dass sich das integrierte Modell insgesamt bewährt hat. Die Nebenbeinutzung lässt sich im Rahmen einzelner theoretischer Ansätze nicht hinreichend erklären. Erst durch das Zusammenspiel verschiedener Einflussfaktoren konnten substanzielle Varianzaufklärungen erzielt werden. Durch die systematische Prüfung von indirekten Effekten wurde zudem verdeutlicht, dass bestimmte Variablen wie Alter oder Fernsehbindung sich zwar nicht direkt, bei genauerer Analyse aber doch indirekt - vermittelt über andere Variablen - auf die Nebenbeinutzung auswirken. Das hier vorgestellte Modell sollte sich auch auf die Nutzung anderer Medienangebote (zum Beispiel Radioprogramme oder Illustrierte) übertragen lassen. Die Ergebnisse sollten ermutigen, die vorhandenen theoretischen Konzepte der Nutzungsforschung stärker aufeinander zu beziehen und soweit möglich zu integrieren, um so zu einem tieferen Verständnis der Mediennutzung zu gelangen. 


\section{Literatur}

Ball-Rokeach, Sandra J. \& DeFleur, Melvin L. (1976): A Dependency Model of Mass-Media Effects. In: Communication Research 3 (1): 3-21.

Becker, Lee B. \& Whitney, D. Charles (1980): Effects of Media Dependencies. Audience Assessment of Government. In: Communication Research 7: 95-120.

Abele-Brehm, Andrea \& Brehm, Walter (1986): Zur Konzeptualisierung und Messung von Befindlichkeit. Die Entwicklung der „Befindlichkeitsskalen“ (BFS). In: Diagnostica 32 (3): 209-228.

Donsbach, Wolfgang \& Tasche, Karl (1999): When Mood Management Fails. A Field Study on the Relationships between Daily Events, Mood, and Television Viewing. Paper presented to the Annual Conference of the International Communication Association (ICA) in San Francisco.

Emmer, Martin; Kuhlmann, Christoph; Vowe, Gerhard \& Wolling, Jens (2002): Der 11. September - Informationsverbreitung, Medienwahl, Anschlusskommunikation. Ergebnisse einer Repräsentativbefragung zu einem Ereignis mit extremem Nachrichtenwert. In: Media Perspektiven (4): 166-177.

Esser, Hartmut (1999): Soziologie: Spezielle Grundlagen. Band 1: Situationslogik und Handeln. Frankfurt/Main: Campus.

Frank, Ronald E. \& Greenberg, Marshall G. (1980): The Public's Use of Television. Beverly Hills, London: Sage.

Gehrau, Volker (2002): Eine Skizze der Rezeptionsforschung in Deutschland. In: Rössler, Patrick; Kubisch, Susanne \& Gehrau, Volker (Hrsg.). Empirische Perspektiven der Rezeptionsforschung. München: Reinhard Fischer, 9-47.

Hasebrink, Uwe (2003): Nutzungsforschung. In: Bentele, Günter; Brosius, Hans Bernd \& Jarren, Otfried (Hrsg.). Öffentliche Kommunikation. Handbuch Kommunikations- und Medienwissenschaft. Wiesbaden: Westdeutscher, 101-127.

Jäckel, Michael (1992): Mediennutzung als Niedrigkostensituation. Anmerkungen zum Nutzenund Belohnungsansatz. In: Medienpsychologie 4: 247-266.

Jäckel, Michael (1997): Wer trägt die Verantwortung? Zur Mediennutzung von Kindern und Jugendlichen. In: Aus Politik und Zeitgeschichte B19-20: 3-12.

Jäckel, Michael \& Wollscheid, Sabine (2004): Medienzeitbudgets im Vergleich. Eine Gegenüberstellung der Langzeitstudie Massenkommunikation und der Zeitbudgeterhebung des statistischen Bundesamtes. In: Medien \& Kommunikationswissenschaft 52 (3): 355-376.

Katz, Elihu; Blumler, Jay G. \& Gurevitch, Michael (1974): Uses of Mass Communication by the Individual. In: Davison, W. Phillips \& Yu, Frederick T. C. (Hrsg.). Mass Communication Research. Major Issues and Future Directions. New York, Washington, London, 11-35.

Kuhlmann, Christoph \& Wolling, Jens (2004): Fernsehen als Nebenbeimedium. Befragungsdaten und Tagebuchdaten im Vergleich. In: Medien \& Kommunikationswissenschaft 52 (3): $386-411$.

McGuire, William J. (1974): Psychological Motives and Communication Gratification. In: Blumler, Jay G. \& Katz, Elihu (Hrsg.). The Uses of Mass Communications. Current Perspectives on Gratifications Research. Beverly Hills, London: Sage, 167-196.

McQuail, Denis; Blumler, Jay G. \& Brown, J. R. (1972): The Television Audience: A Revised Perspective. In: McQuail, Denis (Hrsg.). Sociology Of Mass Communication. Harmondsworth, $135-165$.

Meyen, Michael (2004): Mediennutzung: Mediaforschung, Medienfunktionen, Nutzungsmuster. Konstanz: UVK.

Palmgreen, Philip \& Rayburn, J. D. II (1982): Gratifications Sought and Media Exposure. An Expectancy Value Model. In: Communication Research 9 (4): 561-580.

Palmgreen, Philip; Wenner, Lawrence A. \& Rayburn, J. D. II (1981): Gratification Discrepancies and News Program Choice. In: Communication Research 8 (4): 451-478.

Rheinberg, Falko (2004): Motivation. Stuttgart: Kohlhammer.

Ridder, Christa-Maria et al. (2002): Massenkommunikation VI. Baden-Baden: Nomos.

Rosengren, Karl Erik (1996): Inhaltliche Theorien und formale Modelle in der Forschung über individuelle Mediennutzung. In: Hasebrink, Uwe \& Krotz, Friedrich (Hrsg.). Die Zuschauer als 
Fernsehregisseure? Zum Verständnis individueller Nutzungs- und Rezeptionsmuster. BadenBaden, Hamburg: Nomos.

Rubin, Alan M. (2002): The Uses-and-Gratifications Perspective of Media Effects. In: Bryant, Jennings \& Zillmann, Dolf (Hrsg.). Media Effects. Advances in Theory and Research. Mahwah, New Jersey \& London: Lawrence Earlbaum, 525-548.

Schenk, Michael (2002): Medienwirkungsforschung. 2., vollständig überarbeitete Auflage. Tübingen: Mohr.

Schmitz, Bernhard \& Lewandrowski, Uwe (1993): Trägt das Fernsehen zur Regulierung von Stimmungen bei? Intraindividuelle Analysen zur „Moodmanagement“-Hypothese auf der Grundlage eines dynamisch-transaktionalen Modells. In: Medienpsychologie 5 (1): 64-84.

Vorderer, Peter (1992): Fernsehen als Handlung. Fernsehfilmrezeption aus motivationspsychologischer Perspektive. Berlin: edition sigma.

Vowe, Gerhard \& Wolling, Jens (2001): Die Organisationsfunktion der Netzkommunikation. Wie lassen sich Unterschiede in der Nutzung von Online-Angeboten erklären? In: Maier-Rabler, Ursula \& Latzer, Michael (Hrsg.). Kommunikationskulturen zwischen Kontinuität und Wandel. Universelle Netzwerke für die Zivilgesellschaft. Konstanz: UVK, 269-285.

Vowe, Gerhard \& Wolling, Jens (2004): Radioqualität. Was die Hörer wollen und was die Sender bieten. München: Kopaed.

Wenner, Lawrence A. (1982): Gratifications Sought and Obtained in Program Dependency. A Study of Network Evening News Programs and 60 Minutes. In: Communication Research 9 (4): 539-560.

Wolling, Jens (2004): Qualitätserwartungen, Qualitätswahrnehmungen und die Nutzung von Fernsehserien. Ein Beitrag zur Theorie und Empirie der subjektiven Qualitätsauswahl von Medienangeboten. In: Publizistik 49 (2): 171-193.

Wünsch, Carsten (2001): Musik für jede Stimmungslage. In: Wissenschaftliche Zeitschrift der Technischen Universität Dresden 50 (1/2): 40-46.

Zillmann, Dolf (1994): Über behagende Unterhaltung in unbehagender Medienkultur. In: Bosshart, Louis \& Hoffmann-Riem, Wolfgang (Hrsg.). Medienlust und Mediennutz. Unterhaltung als öffentliche Kommunikation. München, 41-57.

Zillmann, Dolf (2004): Emotionspsychologische Grundlagen. In: Mangold, Roland; Vorderer, Peter \& Bente, Gary (Hrsg.). Lehrbuch der Medienpsychologie. Göttingen u. a.: Hogrefe, 101-128.

Zillmann, Dolf; Hezel, Richard T. \& Medoff, Norman J. (1980): The Effect of Affective States on Selective Exposure to Television Entertainment Fare. In: Journal of Applied Social Psychology 10 (4): 323-329. 